\title{
Análise das Trajetórias de Aprendizagem de Estudantes Inscritos em um MOOC
}

\author{
Napoliana Silva de Souza - souzapoliana2@gmail.com - PPGIE/UFRGS \\ Gabriella Santiago - gabriellasntg@gmail.com - DEG/ UFRGS \\ José Valdeni de Lima - valdeni@ inf.ufrgs.br - PPGIE/ UFRGS \\ Alberto Bastos do Canto Filho - alberto.canto@ufrgs.br - PPGIE/ UFRGS \\ Gabriela Trindade Perry - gabriela.perry@ufrgs.br - PPGIE/ UFRGS
}

\begin{abstract}
Resumo
Os MOOCs representam uma nova forma de acesso ao conhecimento - usualmente aberto e gratuito, alcançando um alto número de inscrições. A partir dessa nova modalidade de distribuição de cursos, surgem novas possibilidades de explorações ligadas à aprendizagem. Neste sentido, essa pesquisa está inserida no âmbito da análise das trajetórias de aprendizagem, e teve como objetivo analisar o comportamento navegacional de inscritos $(n=1415)$ em um MOOC denominado Poesia Grega, a fim de investigar se a trajetória percorrida (linear ou não linear) possui relação com o desempenho dos concluintes $(n=279)$. Para tanto, foram analisados registros de navegação, e os resultados revelaram que a maioria dos estudantes segue uma trajetória não linear. Todavia, o teste de Mann Whitney, para diferença das médias das notas identificou que o grupo que seguiu uma trajetória linear alcançou melhor desempenho se comparado ao grupo não linear.
\end{abstract}

Palavras-chave: MOOCs, trajetórias de aprendizagem, trajetória linear, trajetória não linear.

\section{Analysis of the Learning Paths of Students enrolled in a MOOC}

\begin{abstract}
MOOCs represent a new means of access to knowledge - usually open and free, with large numbers of enrollments. Through this new modality of course distribution new possibilities for investigations of learning experiences emerge. In this sense, this research is inserted in the scope of the analysis of paths learning, the objective of this study was to analyze the navigational behavior of students enrolled $(n=1415)$ in a MOOC called "Greek Poetry", in order to investigate whether the path adhered (linear and nonlinear) is related the performance of the learners who complete the course $(n=279)$. For this purpose, we analyzed the navigation logs of this MOOC, and the results show that most students adhere to a nonlinear path. However, the MannWhitney test used for difference of the means of the grades identified that the group that adhered the linear path achieved better performance when compared to the non-linear group.
\end{abstract}

Keywords: MOOCs, learning trajectories, linear path, non-linear path. 


\section{INTRODUÇÃO}

Os Massive Open Online Courses (MOOCs) surgiram com o propósito de promover a inovação educacional por meio de abordagens pedagógicas que ampliam as possibilidades de aprendizagem, de modo a alcançar um alto número de estudantes (Zheng et al., 2016). Os MOOCs são constituídos de duas dimensões fundamentais: a massividade e a abertura. A massividade está relacionada com a capacidade para atender um número indeterminado de alunos, enquanto a abertura prevê um curso ao alcance de todos, eliminando obstáculos relacionados à localização geográfica, fatores financeiros, ritmos de aprendizagem, tempo de realização do curso (Pilli e Admiraal, 2016).

A estrutura pedagógica dos MOOCs pode ser constituída de materiais diversificados, por exemplo: vídeos, fóruns de discussão, arquivos de texto e com slides (Baturay, 2015). O material produzido para contextos de MOOCs fundamentam-se em "microlearning", adota-se o termo "microlearning" para referir-se as experiências de aprendizagem que incluam o uso de materiais produzidos sob a perspectiva de fragmentação, sendo os módulos e unidades de um curso projetados com base em porções reduzidas de conteúdo, de modo que o estudante possa realizar cada uma das unidades dos materiais educacionais em um tempo estimado de 15 minutos (Sun et al., 2015).

Por meio da variedade de recursos de aprendizagem, e mediante o elevado número de inscritos que os MOOCs alcançam, diversos tipos de dados podem ser coletados e analisados. Os dados de navegação dos usuários podem ser explorados com a finalidade de identificar padrões que emergem durante o percurso, e para o reconhecimento de fatores problemáticos, possibilitando realizar intervenções que contribuam para melhorar o processo de aprendizagem e evitar que ocorra o abandono do curso (Zheng e Yin, 2015; Mukala et al. 2015; Ramos et al., 2015).

A navegação dos estudantes, por sua vez, se conecta as denominadas "trajetórias de aprendizagem", que descrevem os caminhos trilhados por estudantes quando interagem em um ambiente virtual de aprendizagem (Oliveira et al., 2014). Identificar as trajetórias adotadas pelos alunos por meio de análises navegacionais gera resultados que podem ser importantes, contribuindo para a realização de ajustes dos materiais de aprendizagem, adequando-os conforme as características de aprendizagem dos estudantes (Althoff e Fadel, 2016). Desta forma, pode-se considerar que as trajetórias de aprendizagem, além de representar os caminhos seguidos por estudantes, são também um caminho para detectar problemas e meios para aprimorar a aprendizagem (Ramos et al., 2015).

Em contextos de MOOCs, a análise das trajetórias de aprendizagem dos estudantes tem gerado efeitos positivos, sendo utilizadas como meio para identificar percursos de aprendizagem diferenciados, possibilitar a aprendizagem adaptativa, realizando assim uma instrução diferenciada (Tahiri, Bennani e Idrissi, 2017). Nos estudos de Maldonado et al. (2016) e Davis et al. (2016) os autores chamam a atenção para a importância de conhecer os caminhos de aprendizagem dos estudantes de MOOCs e o impacto dos trajetos percorridos na aprendizagem.

Regularmente os MOOCs possuem uma estrutura organizacional em que os conteúdos são disponibilizados em uma ordem de cima para baixo (top-down), e essa forma de organização pode levar à interpretação de uma imposição linear de acompanhamento do material de aprendizagem (Guo e Reinecke, 2014; Zunguze et al. 2017). Considerando esse cenário, esta pesquisa teve como objetivo analisar o comportamento navegacional de alunos inscritos em um MOOC sobre Poesia Grega, a 
fim de investigar se os mesmos acompanham o conteúdo de forma linear ou não linear e se a trajetória percorrida produz efeitos no desempenho em atividades avaliativas.

\section{ANÁLISE DE TRAJETÓRIAS DE NAVEGAÇÃO EM MOOCS}

Maldonado et al. (2016) realizaram um estudo exploratório envolvendo 99 estudantes de um MOOC denominado "Design, Criação e Avaliação de Objetos de Aprendizagem", disponibilizado na plataforma edX. O propósito do trabalho foi analisar o comportamento navegacional de estudantes e identificar se há diferenças na forma de navegação e se os comportamentos navegacionais estão associados aos diferentes estilos de aprendizagem e aos diferentes perfis de autorregulação da aprendizagem. No plano metodológico, os autores utilizaram técnicas de mineração de processos, e além de explorar arquivos de logs os autores aplicaram questionários de autorrelato para identificar qual o estilo de aprendizagem e o perfil de autorregulação de aprendizagem dos participantes. $\mathrm{Na}$ análise de dados, os autores trabalharam com os estilos de aprendizagem segundo a categorização de Alonso, Gallego e Honey (1994): ativo, reflexivo, teórico e pragmático. O perfil de autorregulação considerou os níveis: baixo, médio e alto. Maldonado et al. (2016) mostraram, a partir dos resultados, que estudantes ativos não adotam a sequência linear projetada pelo curso, enquanto estudantes com os estilos de aprendizagem teórico e pragmático seguem a estrutura sequencial do MOOC. Os autores observaram que estudantes com estilo de aprendizagem reflexivo também costumam seguir a sequência estabelecida pelo curso, no entanto, realizam retornos no início dos módulos ao concluírem uma tarefa avaliativa. Outro resultado destacado por Maldonado et al. (2016) revelou que os estudantes que concluem o curso geralmente seguem o caminho sequencial estabelecido pelo MOOC.

Davis et al. (2016) analisaram as trajetórias de aprendizagem a partir dos logs de 113.000 estudantes de quatro MOOCs, hospedados na plataforma edX, os cursos versam sobre: Programação Funcional; Análise de Dados com Phyton; Framing; Inovação Responsável. A pesquisa foi guiada pelo seguinte questionamento: "Em que medida os alunos aderem ao caminho de aprendizagem projetado pelo MOOC?". Os autores examinaram se os estudantes seguem o caminho proposto pelo curso ou efetuam desvios de caminhos, bem como analisaram se o sucesso e insucesso de um aluno tem relação com o caminho percorrido. Os seguintes elementos foram analisados: vídeos, questionários, páginas de progresso e fóruns de discussão, em busca de comportamentos diferenciados, bem como padrões comportamentais. Em uma perspectiva geral, os principais resultados apontados pelos autores evidenciaram que estudantes de sucesso (aqueles que são aprovados na avaliação final, obtendo certificado) efetuam menos desvios de caminhos se comparados aos estudantes não aprovados. Todavia, no que se refere especificamente aos vídeos, os autores notaram que os estudantes aprovados e não aprovados possuem comportamentos de ignorar vídeos, além disso, observaram que o mecanismo de avançar dos vídeos é utilizado com mais frequência pelos alunos do que o recurso retroceder.

Quon e Pong (2016) analisaram o percurso de aprendizagem de estudantes inscritos em um MOOC sobre programação em Java disponibilizado na plataforma edX, com a intenção de descobrir os caminhos de aprendizagem que os estudantes seguem. Os autores não mencionaram a quantidade de alunos que integraram a amostra de análise. Quon e Pong (2016) observaram uma série de variáveis tais como: idade, proficiência em inglês; experiência prévia com o assunto do curso; experiência com MOOCs, desempenho, entre outros elementos. Os autores ressaltam que foram analisados dados semanais, que contabilizaram o envio de tarefas, postagens em fóruns, 
cliques em vídeos. A partir dos resultados Quon e Pong (2016) relataram a identificação de 140 percursos de aprendizagem diferenciados. Além disso, os autores encontraram relações entre as experiências e conhecimentos prévios dos estudantes com o percurso que o estudante segue, e também observaram ocorrências em que estudantes com níveis diferenciados de proficiência linguística (proficiência básica, intermediário, proficiente, avançado) concluíram todos os módulos do MOOC, obtendo um ótimo desempenho e ocasiões em que estudantes com baixo nível de proficiência obtiveram desempenho inferior se comparado com os participantes que seguiram o mesmo percurso de aprendizagem.

$\mathrm{Na}$ pesquisa de Guo e Reinecke (2014) foi analisado o comportamento navegacional de 140.546 estudantes de 196 países inscritos em quatro MOOCs armazenados na plataforma edX, a fim de identificar diferenças de navegação/interação com os recursos de aprendizagem de MOOCs. Os MOOCs abordaram conteúdos diversificados, incluindo as seguintes temáticas: Introdução a Ciência da Computação \& Programação; Inteligência Artificial; Química; Estatística para a Saúde Pública. O estudo foi guiado pela hipótese de que o comportamento navegacional dos estudantes pode ser diferente dependendo da idade e do país de origem dos participantes. A partir dos resultados os autores descobriram que a maioria dos estudantes navega pelos recursos de aprendizagem de MOOCs de forma não linear. Guo e Reinecke (2014) confirmaram a existência de comportamentos de navegação diferenciados em virtude das variadas características demográficas dos participantes. Por meio dos resultados, os autores relataram que estudantes mais velhos possuem mais autonomia e aderem mais ao trajeto não linear se comparado aos alunos mais jovens. Além disso, Guo e Reinecke (2014) identificaram que os estudantes mais jovens, assim como os participantes da Índia, Quênia e Paquistão aderem em maior proporção à estrutura de aprendizagem estabelecida pelo professor se comparados aos participantes dos Estados Unidos e dos países europeus.

Por meio dos estudos supracitados é possível observar que os trabalhos estão direcionados para a avaliação da correlação entre as trajetórias de aprendizagem de estudantes de MOOCs e outras variáveis, a fim de buscar um padrão que explique - e permita prever - comportamentos de navegação e desempenho. Com relação à diferença entre as pesquisas mencionadas e a proposta deste artigo, destaca-se que os trabalhos citados analisaram dados de cursos de plataformas de MOOCs internacionais, acessadas por usuários que falam diversos idiomas, e no contexto deste trabalho o objeto de análise corresponde a um MOOC disponível em uma plataforma brasileira de cursos online. Outro aspecto divergente refere-se à análise dos dados, nesta pesquisa é observado se o caminho percorrido por estudantes tem impacto nas notas obtidas em tarefas.

\section{PROCEDIMENTOS METODOLÓGICOS}

Essa pesquisa consistiu em um estudo de caso de um MOOC disponibilizado na plataforma Lúmina, que pertence à Universidade Federal do Rio Grande do Sul. Os MOOCs disponíveis no Lúmina mantêm registros de navegação, ou arquivos de log. Segundo Azevedo et al. (2013) esta é uma prática usual. Em seu termo de uso ${ }^{1}$, o Lúmina informa a realização de coleta de dados de navegação.

Nesse contexto, a análise das trajetórias de aprendizagem de estudantes de MOOCs foi realizada observando-se os trajetos segundo duas categorias: "trajetória linear" e "trajetória não linear". A trajetória linear compreende o trajeto em que o estudante acessa os conteúdos de maneira sequencial, seguindo a ordem pré-definida

${ }^{1}$ https://lumina.ufrgs.br/faq/termo_de_uso_Lumina.pdf 
pelo MOOC. A trajetória não linear refere-se ao trajeto não ordenado, isto é, o estudante acessa os conteúdos sem obedecer à estrutura sequencial sugerida pelo MOOC. O objetivo da análise das trajetórias foi responder às seguintes questões de pesquisa:

Questão de Pesquisa 1. Qual a forma de navegação que os estudantes aderem ao participar de um MOOC?

Questão de Pesquisa 2. O percurso de aprendizagem adotado por estudantes influencia no desempenho obtido em tarefas avaliativas realizadas no curso?

\subsection{Objeto de Análise}

O objeto de estudo analisado foi um MOOC denominado "Poesia Grega", que contabilizava 1415 inscritos em maio de 2018. Trata-se de um curso on-line desenvolvido com o propósito de abordar gêneros poéticos gregos, incorporando conteúdos focalizados na Poesia Épica e Poesia Lírica. A descrição do curso o define como de nível "básico", e estimativa total de tempo de estudo é de 14 horas.

O MOOC é composto por 15 vídeos, 4 textos complementares e 1 questionário de avaliação (com 5 questões). Possui, como todos os MOOCs do Lúmina, um vídeo e um texto de apresentação, uma pesquisa do perfil do estudante (que coleta dados de forma anônima), um fórum de dúvidas e sugestões e uma pesquisa (anônima) de avaliação do curso.

\subsection{Perfil da Amostra}

Os dados do arquivo de log abrangem todo o período de oferta do curso - de agosto de 2017 até hoje (maio de 2018) - contudo, o intervalo que possui concluintes vai de 02/09/ 2017 a 28/04/2018. O curso possuía (em maio de 2018) 1415 inscritos, sendo que 279 concluíram o curso em sua totalidade. Destes, 159 identificam-se com o gênero feminino e 120 com o gênero masculino. Em relação ao nível instrucional, 35 estão cursando ou concluíram o ensino médio; 111 estão cursando o ensino superior e 132 possuem um curso superior.

\subsection{Identificação das Trajetórias Percorridas}

Os procedimentos para detectar as trajetórias de aprendizagem foram realizados por meio dos logs Geral e de Notas do Lúmina, que utilizam o formato csv (comma separeted value), de forma que a importação para a ferramenta Microsoft Excel e a aplicação de filtros foi simplificada.

No log Geral do curso, cada estudante estava representado por uma linha. O banco de dados possuía, além da coluna nome, uma coluna para cada uma das 20 atividades, contendo sua data de conclusão no formato DD/MM/AAAA hh:mm:ss (que ficava vazia caso a atividade não tivesse sido concluída). Para excluir da população os estudantes que não haviam completado todas as atividades do curso, aplicou-se um filtro a cada uma das colunas, de forma a selecionar apenas as atividades com as datas diferentes de "vazio".

O segundo passo foi importar os dados do log de Notas, que possuía uma coluna para o nome, uma para a média (calculada pelo Lúmina) e uma para o número de tentativas utilizadas. Escolheu-se a nota relativa à primeira tentativa, pois o Lúmina (à semelhança de outras plataformas) informa o gabarito dos testes após a submissão das respostas.

O terceiro passo foi alinhar os dois bancos, de forma a mostrar a nota de cada aluno concluinte e as datas de realização de cada atividade na mesma planilha. Essa parte foi 
realizada manualmente, copiando e colando os dados do log Notas na planilha dos valores do log Geral, pois o Lúmina não agrega os dois dados.

A análise de dados a respeito das trajetórias foi realizada considerando que um estudante que percorre uma trajetória linear realiza as atividades na sequência indicada na página do curso. Sendo assim, ao subtrair-se a data da atividade $A_{n}$ da atividade $A_{(n}{ }^{-}$ 1), o resultado deveria ser sempre positivo e maior que zero. Partindo deste princípio, para cada aluno, foram contadas as ocorrências do tipo $A_{n}-A_{(n-1)}>0$, em cada uma das 20 colunas das atividades do curso. Valores iguais a 0 são possíveis apenas se o aluno realizou as duas atividades ao mesmo tempo. Valores menores que 0 significam que 0 aluno realizou a atividade $A_{(n-1)}$ antes da atividade $A_{n}$. A Figura 1 mostra um excerto desta tabela.

\begin{tabular}{c|c|c|c|c|c|c|c} 
Notas & lliada & Odisseia & Saro & Safo Fr.1 & Safo Fr.31 & Saro Fr.102 & SaroFr.1688 \\
\hline 6 & 0,0041666667 & 0,004166667 & 0,006944444 & 0,0069444444 & 0,009027778 & 0,014583333 & 0,002083333 \\
\hline 10 & 0,010416667 & 0,003472222 & 0,003472222 & 0,029166667 & 0,021527778 & 6,5125 & 0,004861111 \\
\hline 10 & 0,003472222 & 0,004861111 & 0,002083333 & 0,004166667 & 0,020138889 & 0,029861111 & 0,001388889 \\
\hline 8 & 7,799305556 & 5,20625 & 0,002083333 & 0,00625 & 12,98402778 & 0,008333333 & 0,006944444 \\
\hline 8 & 0,004166667 & 0,006944444 & 0,004166667 & 0,00625 & 0,002777778 & 0,002083333 & 0,000694444 \\
\hline 10 & 0,000694444 & 0,000694444 & 0,005555556 & 5,291666667 & 0,007638889 & 0,004166667 & 0,002777778 \\
\hline 8 & 0,1625 & 0,88888889 & 0,002083333 & 0,011805556 & 2,838194444 & 0,003472222 & 0,001388889 \\
\hline 10 & 2,638888889 & 0,002083333 & 0,013194444 & 0,011805556 & 0,004166667 & 0,005555556 & 0,002083333 \\
\hline
\end{tabular}

Figura 1 - Excerto da tabela de logs e notas, indicando a temporalidade da realização das atividades.

O último passo foi ordenar os resultados a partir da coluna da contagem das ocorrências de valores maiores que zero e colar os dados das notas no SPSS, agrupados por categoria (linear ou não linear).

\section{RESULTADOS}

Os resultados são abordados em duas perspectivas: em relação às trajetórias que o grupo de concluintes realizou e o teste de hipótese para diferença das médias.

\subsection{Trajetória Seguida pelos Estudantes}

A Figura 2 mostra os percursos dos concluintes (um por linha). Nela pode-se observar que a maioria dos concluintes segue trajetórias que alternam sequências temporais lineares (atividade A1 realizada antes da atividade A2). Notou-se (e marcouse em cinza) uma área que percorre 4 colunas, que mostra uma grande quantidade de espaços em branco - identificados na legenda como atividades que foram realizadas em sequência cronológica não-linear. Estes materiais correspondem a um módulo do curso nomeado "Materiais Complementares", composto por 4 textos. Analisando esta figura, concluiu-se que os estudantes não consultaram este material apenas ao final do curso, de forma que se atribui a isso a maior parcela do motivo do não seguimento da trajetória linear. Sendo assim, acredita-se que possa ter havido diferenças entre a adoção de trajetórias lineares e trajetórias não lineares, caso sejam desconsideradas as datas de leitura dos materiais complementares. 


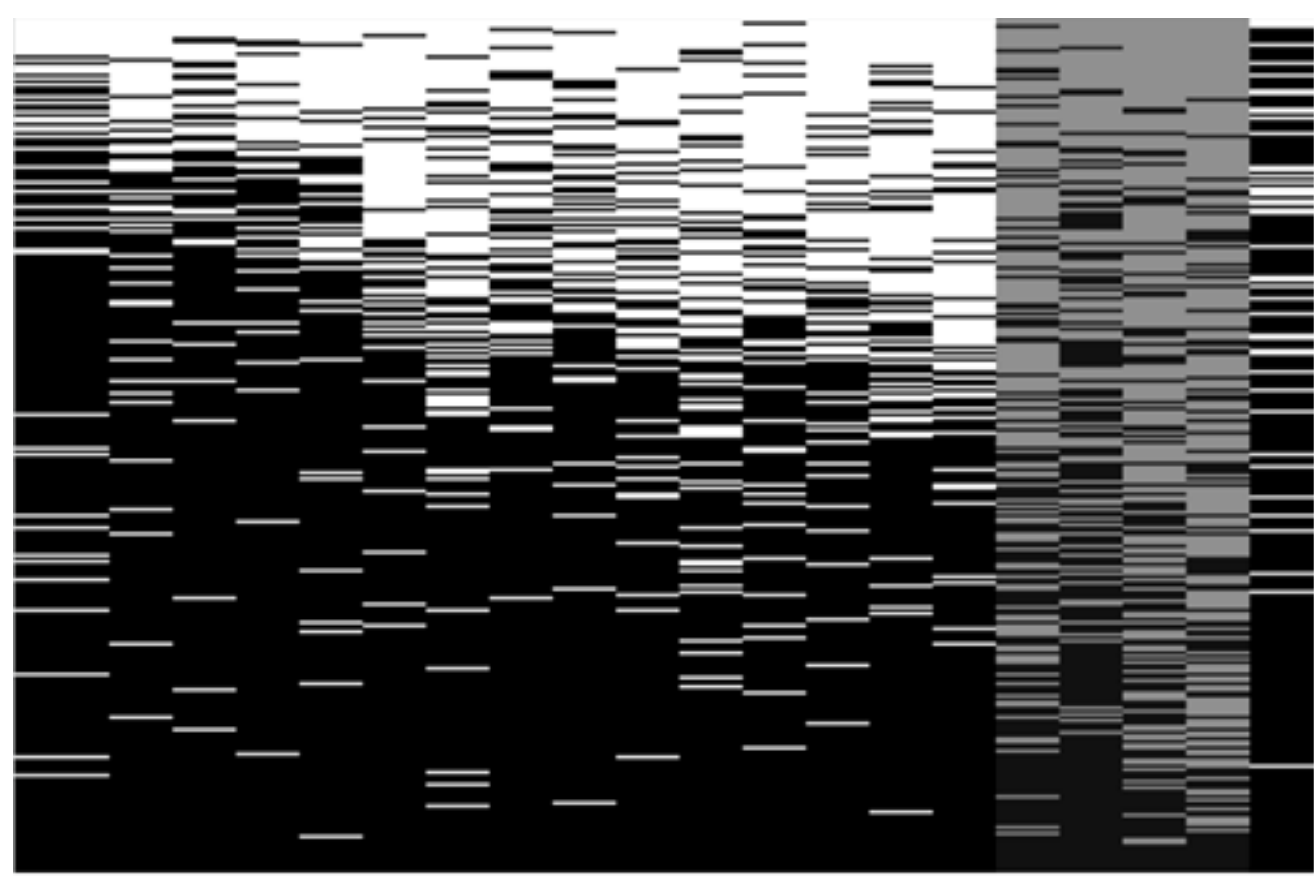

Atividade realizada na sequência cronológica linear

Atividade realizada na sequência cronológica não linear

Figura 2- Trajetórias dos concluintes do curso de Poesia Grega.

Quando se consideram todos os materiais, dentre os 279 estudantes concluintes, apenas 9 realizaram uma trajetória perfeitamente linear, sem qualquer desvio na sequência indicada no curso. Como poucos alunos seguiram trajetórias perfeitamente lineares, decidiu-se ampliar a classificação para abarcar alunos que tivessem percorrido no mínimo $90 \%$ do curso na sequência indicada pelo professor (no máximo 2 atividades fora de ordem). Dessa forma, esse grupo foi ampliado para 76 pessoas. Os 203 estudantes restantes seguiram trajetórias não lineares.

Ao desconsiderar as datas de leitura dos textos dos materiais complementares, encontrou-se que 141 estudantes seguiram trajetórias não lineares, enquanto 138 seguiram trajetórias lineares. A Tabela 1 sumariza estes dados.

Tabela 1 - Quantidades de estudantes por tipo de trajetória e material instrucional

\begin{tabular}{lcc}
\hline & $\begin{array}{c}\text { Trajetória não } \\
\text { linear }\end{array}$ & Trajetória linear \\
\hline Sem materiais complementares & 141 & 138 \\
\hline Com materiais complementares & 203 & 76
\end{tabular}

Desta forma, não responde-se à primeira questão de pesquisa: Qual a forma de navegação que os estudantes aderem ao participar de um MOOC? de forma conclusiva, pois ainda que a maior parte dos estudantes siga trajetórias não lineares, quando se desconsidera as datas de leitura dos materiais complementares, a diferença é muito reduzida. Este fato chamou a atenção, e precisa ser melhor investigado.

\subsection{Desempenho obtido pelos participantes}

Para o teste de hipóteses da diferença entre as notas dos estudantes que seguiram trajetória linear e não linear, tanto para o cenário considerando os materiais complementares como não os considerando, foi utilizado o teste de Mann Whitney, com 
$\alpha=0,05$ (nível de certeza de 95\%). O motivo é que as notas não representam uma variável normalmente distribuída - o teste de Shapiro-Wilk, resultou em $p=0.001$, valor menor que o critério de decisão para aceitar a hipótese da normalidade da variável: 0.005. Para essa finalidade duas hipóteses foram enunciadas:

- Hipótese nula, ou $\mathbf{H}_{\mathbf{0}}$ As notas de quem segue a trajetória linear são menores ou iguais de quem não segue.

- Hipótese alternativa, ou $\mathbf{H}_{1}$ As notas de quem segue a trajetória linear são maiores de quem não segue.

Para a comparação das notas dos estudantes, sem considerar os materiais complementares, o teste de Mann-Whitney indicou que as notas do grupo que seguiu a trajetória linear foram maiores (média de 7,82) que as dos que seguiram a trajetória não linear (média de 6,63), com $U=7472.5$ e $\mathrm{p}=0,001$. Para a comparação das notas dos estudantes, considerando os materiais complementares, o teste de Mann-Whitney indicou que as notas do grupo que seguiu a trajetória linear também foram maiores (média de 8,02) que as dos que seguiram a trajetória não linear (média de 6,921), com U $=5897$ e $\mathrm{p}=0,001$, o que implica a rejeição da hipótese nula. A Tabela 2 sumariza os dados referentes aos cenários com e sem a análise das datas de conclusão das leituras dos materiais complementares.

Tabela 2 - Estatísticas descritivas das amostras

\begin{tabular}{llccc}
\hline \multirow{2}{*}{ Sem materiais complementares } & Média ranqueada & $\mathrm{n}$ & Média \\
& $\begin{array}{c}\text { Trajetória } \\
\text { não linear }\end{array}$ & 124 & 141 & 6,63 \\
\cline { 2 - 5 } & $\begin{array}{c}\text { Trajetória } \\
\text { linear }\end{array}$ & 156,35 & 138 & 7,82 \\
\hline \multirow{2}{*}{ Com materiais complementares } & $\begin{array}{l}\text { Trajetória } \\
\text { não linear }\end{array}$ & 131 & 203 & 6,921 \\
\cline { 2 - 5 } & $\begin{array}{c}\text { Trajetória } \\
\text { linear }\end{array}$ & 163,9 & 76 & 8,02
\end{tabular}

Com o objetivo de encontrar uma explicação para este resultado, considerou-se que possa existir uma relação entre o nível educacional dos concluintes com o tipo de trajetória seguida - considerou-se que essa hipótese tem maior poder de explicação do que gênero ou faixa etária. Sendo assim, investigou-se a hipótese da independência entre a quantidade de concluintes em relação ao tipo de trajetória e nível instrucional.

- Hipótese nula, ou $\mathbf{H}_{0}$ As quantidades de concluintes que seguem determinado tipo de trajetória não têm relação com as quantidades de concluintes com determinado nível instrucional.

- Hipótese alternativa, ou $\mathbf{H}_{1}$ As quantidades de concluintes que seguem determinado tipo de trajetória estão relacionadas com as quantidades de concluintes com determinado nível instrucional.

Para responder a esta pergunta, foi realizado o teste do Chi Quadrado, que pode ser utilizado quando as variáveis em análise são nominais e quando os valores têm a mesma probabilidade de ocorrer em cada uma das categorias. Este teste avalia se as ocorrências dentro de cada categoria estão dentro do esperado caso sejam verdadeiramente aleatórias, ou se há alguma relação entre elas (hipótese alternativa). A tabela 3 traz os dados da tabela de contingência, necessária para avaliar o resultado deste teste $(\alpha=0,05)$. Quando não se consideram as datas dos materiais complementares, o valor do $\chi^{2}$ é 6,7 o 
que resulta num $\mathrm{p}=0,03$ - o que implica a rejeição da hipótese nula. Este resultado indica haver uma relação entre o nível instrucional e o tipo de trajetória que explicaria as ocorrências em cada uma das categorias, para além do mero acaso.

Tabela 3 - tabela de contingência para o cenário sem as datas dos materiais complementares

\begin{tabular}{rccccc}
\hline & \multicolumn{3}{c}{ Trajetória não linear } & \multicolumn{3}{c}{ Trajetória linear } & \\
\cline { 2 - 6 } & Observado & Esperado & Observado & Esperado & $\sum$ \\
\hline Ensino médio & 25 & 17.69 & 10 & 17.31 & 35 \\
\hline Ensino superior incompleto & 53 & 56.10 & 58 & 54.90 & 111 \\
\hline Ensino superior completo & 63 & 67.22 & 70 & 65.78 & 133 \\
\hline$\Sigma$ & 141 & & 138 & & 279
\end{tabular}

Na tabela 3 pode-se ver que a quantidade de pessoas que tem ensino médio é menor que o esperado, assim como a quantidade de pessoas com curso superior (completo e incompleto) é maior que o esperado. Sendo assim, conclui-se que pessoas com nível instrucional alto tenderam, neste curso, a utilizar trajetórias lineares.

No entanto, ao incluir as datas de conclusão das atividades complementares, a hipótese nula não pode ser rejeitada, pois a diferença entre as frequências esperada e observada foram pequenas - o que significa que a quantidade de ocorrências em cada categoria pode ser explicada apenas pelo mero acaso. A tabela 4 traz os dados da tabela de contingência, necessária para avaliar o resultado deste teste $(\alpha=0,05)$. O valor do $\chi^{2}$ é 3,4 o que resulta num $\mathrm{p}=0,18$ - o que implica a aceitação da hipótese nula.

Tabela 4 - tabela de contingência para cenário com as datas dos materiais complementares

\begin{tabular}{rccccc}
\hline & \multicolumn{3}{c}{ Trajetória não linear } & \multicolumn{3}{c}{ Trajetória linear } & \\
\cline { 2 - 6 } & Observado & Esperado & Observado & Esperado & $\sum$ \\
\hline Ensino médio & 30 & 25,47 & 5 & 9,53 & 35 \\
\hline Ensino superior incompleto & 78 & 80,76 & 33 & 30,24 & 111 \\
\hline Ensino superior completo & 95 & 96,77 & 38 & 36,23 & 133 \\
\hline$\Sigma$ & 203 & & 76 & & 279
\end{tabular}

\section{CONCLUSÃO}

As conclusões deste artigo estão atreladas às definições dos conceitos utilizados na análise: concluintes e trajetórias. Consideraram-se como concluintes apenas os alunos que realizaram todas as atividades do curso. Contudo, há que se ressaltar que não é possível saber até que ponto um determinado aluno aproveitou de forma efetiva os materiais de cada atividade, pois o único controle que a plataforma oferece é a data de acesso. Caso um aluno tenha aberto os materiais das atividades em abas diferentes de seu navegador web, o Lúmina irá considerar que este aluno concluiu as atividades na mesma data. Como relatado nos Resultados, percebemos que vários estudantes apresentaram este comportamento, o que não pode ser contornado pelas ferramentas de análise. Esta situação se estende para todas as plataformas de MOOCs, haja visto não ser possível afirmar com certeza se um texto foi lido integralmente ou se um vídeo foi assistido até o final (com atenção).

A definição que usamos para o conceito de trajetória também tem um impacto nos resultados encontrados. Há mais de uma forma de descrever e modelar trajetórias, e a escolha feita nesse artigo considera somente a existência de uma diferença positiva entre as datas de duas atividades que deveriam ser realizadas consecutivamente. Este método 
não considera, por exemplo, se as atividades foram realizadas no momento certo, isto é, se a atividade $A_{1}$ foi realizada em $t_{1}$. Por este motivo, pretende-se expandir a análises dos logs dos cursos do Lúmina para abarcar outros modelos de trajetórias.

$\mathrm{O}$ fato de não termos relacionado as notas e as trajetórias com outras variáveis independentes, como a capacidade de autorregulação, à semelhança de Maldonado et al. (2016) é uma limitação desta pesquisa. No entanto, a variável nível instrucional apontou uma associação positiva com o tipo de trajetória escolhida, porém apenas quando não se considera a conclusão das atividades complementares na análise. Nesse caso, observouse que estudantes que estão matriculados ou graduaram-se em cursos de nível superior tendem a adotar trajetórias lineares. Todavia, ao incluir os materiais complementares na análise, esta relação não se verifica. Conclui-se que os materiais complementares não são consumidos apenas no final do curso, o que reforça ainda mais a necessidade de explorar diferentes tipos de trajetórias para obter mais elementos para compreender a forma como os estudantes navegam nos MOOCs.

Contudo, o fato de as notas dos estudantes que seguem trajetórias lineares serem maiores que a dos estudantes que não seguem é um resultado relevante. Considerando a possibilidade que este MOOC (Poesia Grega) reúne conteúdos com conceitos que podem estar interligados, os resultados obtidos nesta pesquisa sugerem que os alunos que adotaram a trajetória linear podem ter alcançado melhor desempenho por seguirem sequencialmente os conteúdos conforme a estrutura pré-definida pelo MOOC.

\section{AGRADECIMENTOS}

Agradecemos o apoio financeiro da Capes, à SEAD | UFRGS - Secretaria de Educação à Distância, pelo suporte incondicional à plataforma Lúmina, ao professor Leonardo Antunes, do MOOC Poesia Grega, e aos colegas Lucília Donato, Maria Angélica, Igor Kuhn, Giovanni Machado e Luis Segovia pelas sugestões.

\section{REFERÊNCIAS BIBLIOGRÁFICAS}

Alonso, C.; Gallego, D.; Honey, P. Los Estilos de Aprendizaje, Procedimiento de Diagnostico y Mejora. Ediciones Mensajero. Universidad de Deusto. Bilbao, 1994.

ALTHOFF, A. E.; FADEL, L.Visualização da trajetória de navegação de usuários em ambiente virtual de aprendizagem. Revista Brasileira de Design da Informação, vol. 17, n 1, p. 46-66, 2016.

AZEVEDO, R.; HARLEY, J.; TREVORS, G.; FEYZI-BEHNAGH, R.; DUFFY, M.; BOUCHET, F.; LANDIS, R.S. Using trace data to examine the complex roles of cognitive, metacognitive, and emotional self-regulatory processes during learning with multi-agent systems. In: R. Azevedo \& V. Aleven (Eds.), International handbook of metacognition and learning technologies, vol. 26, p. 427-449, 2013.

BATURAY, M. H. An overview of the world of MOOCs. Procedia - Social and Behavioral Sciences, vol. 174, p. 427-433, 2015.

DAVIS, D.; CHEN, G.; HAUFF, C.; HOUBEN, G. J. Gauging MOOC Learners' Adherence to the Designed Learning Path. In: EDM '16: 9th International Conference on Educational Data Mining, p. 54-61, 2016.

GUO, P. J.; REINECKE, K. Demographic Differences in How Students Navigate Through MOOCs.In: Proceedings of the First ACM Conference on Learning @ Scale Conference (L@S ’14), p. 21-30, 2014. 
MALDONADO, J.J.; PALTA, R.; VÁZQUEZ, J.; BERMEO, J.L.; PÉREZSANAGUSTÍN, M.; MUNOZ-GAMA, J. Exploring differences in how learners navigate in MOOCs based on self-regulated learning and learning styles: A process mining approach. In: XLII Latin American Computing Conference (CLEI), p. 1-12, 2016.

MUKALA, P., BUIJS, J.; LEEMANS., M.; VAN DER AALST, W. Exploring students' learning behaviour in moocs using process mining techniques. Tech. rep., Technische Universiteit Eindhoven, p. 1-26, 2015.

OLIVEIRA, J. P. M.; LIMA, J. V.; WIVES, L. K.; PERNAS, A. M.; GASPARINI, I.; FERNÁNDEZ, A.; DÍAZ, A. Adaptatividade Geocultural em Ambientes Virtuais de Aprendizagem. RIED, vol. 17, $\mathrm{n}^{\circ}$ 1, p. 83-109, 2014.

PILLI, O.; ADMIRAAL, W.A Taxonomy of Massive Open Online Courses. Contemporary Educational Technology, vol. 7, n 3, p. 223-240, 2016.

QUON, A.; PONG, TING-CHUEN. Learning Pathways Amongst Diverse Students in a Massive Open Online Course. In: Proceedings of the Third International Conference on Learning with MOOCs (LWMOOCs'16), p. 71-73, 2016.

RAMOS, D.; OLIVEIRA, E.; MONTEVERDE, I.; OLIVEIRA, K. Trilhas de Aprendizagem em Ambientes Virtuais de Ensino-aprendizagem: Uma Revisão Sistemática da Literatura. In: XXVI Simpósio Brasileiro de Informática na Educação (SBIE 2015), p. 338-347, 2015.

SUN, G.; CUI, T.; GUO, W.; BEYDOUN, G.; XU, D.; SHEN, J. MicroLearning Adaptation in MOOC: a Software as a Service and a Personalized Learner Model. In: the 14th International Conference on Web-based Learning (ICWL), p.174-184, 2015.

TAHIRI, J. S.; BENNANI, S.; IDRISSI, M. K.diffMOOC: Differentiated Learning Paths Through the Use of Differentiated Instruction within MOOC. In: International Journal of Emerging Technologies in Learning, vol. 12, $\mathrm{n}^{\circ}$ 3, p. 197- 218, 2017.

ZHENG, S.; WISNIEWSKI, P.; ROSSON, M. B.; CARROLL, J. M. Ask the Instructors: Motivations and Challenges of Teaching Massive Open Online Courses. In: Proceeding CSCW '16. Proceedings of the 19th ACM Conference on Computer Supported Cooperative Work \& Social Computing, p. 206-221, 2016.

ZHENG, Y; YIN, B. Big Data Analytics in MOOCs. In: 2015 IEEE International Conference on Computer and Information Technology; Ubiquitous Computing and Communications, p. 681- 686, 2015.

ZUNGUZE, M. C.; NUNES, F. B.; HANNEL, K.; FRANCO, S. R. K.; LIMA, J. V. Relação entre Estilos de Aprendizagem e forma de navegação em Apresentações Paralelas Multimídia. Informática na Educação: teoria \& prática, vol.20, n 1, 2017. 